\title{
Characterization of Purified Coconut Oil Bodies as an Encapsulating Agent for Doxorubicin and Paclitaxel
}

\author{
Pamela T. Aliman, ${ }^{1}$ Ronina Franne N. Cada, ${ }^{1}$ Mark Kevin P. Devanadera, MSc, ${ }^{1,2}$ \\ Alexis M. Labrador, MSc ${ }^{1}$ and Myla R. Santiago-Bautista, MSc ${ }^{1,2,3}$ \\ ${ }^{1}$ Department of Biochemistry, Faculty of Pharmacy, University of Santo Tomas, Manila, Philippines \\ ${ }^{2}$ Research Center for Natural and Applied Sciences, University of Santo Tomas, Manila, Philippines \\ ${ }^{3}$ The Graduate School, University of Santo Tomas, Manila, Philippines
}

\begin{abstract}
Introduction. Doxorubicin (DOX) and paclitaxel (PTX) are both widely used anticancer drugs with a broad spectrum of antitumor activity, commonly against breast, ovarian, and lung cancers. Currently, these drugs are commercially available in liposomal formulations for their use in chemotherapy. This study generally proposed coconut oil bodies (COB) obtained from Cocos nucifera $\mathrm{L}$. as an alternative carrier for DOX and PTX rather than the currently used liposome.
\end{abstract}

Objectives. This study aimed to compare standard liposome and coconut oil bodies as drug carriers in terms of their microencapsulation efficiencies, lipid profiles, in vitro drug release and stability, as well as their cholesterol levels.

Methods. Coconut oil bodies (COB) were isolated and purified from Cocos nucifera L. by modified sucrose gradient method followed by microencapsulation of standard drugs (doxorubicin and paclitaxel) through selfassembly and freeze-thaw method. The two standard drugs were encapsulated using COB and standard liposome. Encapsulation efficiency of both materials were determined. Lipid profiles of both encapsulating materials were analyzed by Fourier-transform infrared spectroscopy, gas chromatography-flame ionization detector, and cholesterol level determination. In vitro drug release and $\mathrm{pH}$ stability of both encapsulated drugs were analyzed.

Results. Doxorubicin (DOX) and paclitaxel (PTX) were successfully incorporated in COB. Lauric acid was mainly abundant in COB and was able to lower cholesterol levels $(5 \mathrm{mg} / \mathrm{dL})$. COB incorporated with DOX and PTX showed stability at acidic and neutral $\mathrm{pH}$. Drug release profile showed a rapid outburst within 3 hours compared to liposome encapsulated DOX and PTX.

Conclusion. Our study showed the encouraging potentials of using COB as wall materials that will make them attractive candidates for the formulation of pharmaceuticals for optimized drug delivery of cancer chemotherapeutics DOX and PTX.

Key Words: coconut oil bodies, liposome, microencapsulation, doxorubicin, paclitaxel, Cocos nucifera L

\section{INTRODUCTION}

Paper won $2^{\text {nd }}$ place and presented at the $2^{\text {nd }}$ International Symposium and $9^{\text {th }}$ Annual Scientific Conference by Metro Manila Health Research and Development Conference (MMHRDC), May 24-25, 2018, Pan Pacific Hotel Manila.

Corresponding author: Myla R. Santiago-Bautista, LPT, MSc Department of Biochemistry

Faculty of Pharmacy

Research Center for Natural and Applied Sciences

University of Santo Tomas

Manila 1015, Philippines

Email: mrsantiago@ust.edu.ph
Chemotherapy has been the main modality of treatment for cancer patients. However, challenges such as inadequate accessibility of drugs to the tumor tissue, intolerable toxicity, development of multi-drug resistance, and the dynamic heterogeneous biology of the growing tumors limit the success rate of cancer chemotherapeutics. ${ }^{1}$ Among the several types of chemotherapeutic drugs, doxorubicin (Adriamycin) and paclitaxel (Taxol) are two of the first-line treatments used for advanced and metastatic cancers. ${ }^{2}$ Doxorubicin (DOX) is an antibiotic derived from Streptomyces peucetius and is part of the anthracycline class of chemotherapeutic agents (Figure 1). It is mainly used for the treatment of soft 
tissue and bone sarcomas as well as cancers of the breast, ovary, bladder, and thyroid. ${ }^{3}$ Treatment with doxorubicin is accompanied with high risk of cardiotoxicities and other side effects such as myelosuppression, alopecia, severe acute nausea and vomiting, and mucositis. ${ }^{4}$ Paclitaxel (PTX) is an effective antineoplastic agent and has a wide spectrum of antitumor activity, particularly against ovarian, breast, and lung cancers. However, similar to doxorubicin, the delivery of paclitaxel is also associated with significant challenges. Being highly lipophilic and lacking ionizable functional groups is the drug's major limitations causing difficulties in formulation as well as clinical problems. ${ }^{5}$

These hindrances limit the therapeutic potential of both drugs in clinical use. An approach to solve such problems is the use of drug carriers. Numerous delivery systems are continuously being explored for further development. Drug delivery systems are means of administering a pharmaceutical compound to achieve therapeutic effect and several studies have already been conducted in the formulation of encapsulated drugs. These include use of liposomes, proliposomes, microspheres, gels, prodrugs, and cyclodextrins. ${ }^{6}$

Microencapsulation is the technology of packaging solid, liquid, or gaseous material within thin polymeric coatings, forming small particles called microcapsules. The coating serves as a protective film to isolate the active substance or the core and allows its release in the ideal place or ideal time. This technology has gained importance in various areas such as pharmaceutics, food, agriculture, and medicine given the value of its use and advantages. ${ }^{7}$ As drug delivery systems, these microcapsules give material structuration, protection, controlled release of the encapsulated active agents, and more. ${ }^{8}$

Currently, liposomal preparations are so far the most used method in microencapsulation technology. Currently, both doxorubicin and paclitaxel are already commercially available as liposome-encapsulated drugs - Doxil $^{\circledR}$ and Lipusu $^{\circledR}$, respectively. Liposomes are self-assembled spherical vesicles with one or more phospholipid bilayers separating the inner aqueous environment from the outer aqueous medium. ${ }^{9}$ Liposomes may provide advantages, including biocompatibility, capacity for self-assembly, and the ability to carry large payloads. ${ }^{10}$

Although the clinical use of liposome brought significant advances in the formulation of these drugs, there are limitations which still needs further optimization. In the early stages of development of liposomal formulations, several problems associated with the use of liposomes were encountered such as a rapid reticuloendothelial system (RES) clearance and triggering of the innate immune system. ${ }^{11}$ These challenges that come with the use of liposomes still hinder the therapeutic potential of both drugs, doxorubicin and paclitaxel, despite resolving the problems experienced with the drugs alone. Clearly, there are still much needed improvement in the formulation of the liposome as coatings and great effort must still be exerted in looking for an appropriate drug vehicle for both doxorubicin and paclitaxel.
Searching for alternative wall materials that could carry the anticancer drug can be another solution. The use of oil bodies as a stable biological microencapsulant offers a strategy in carrying active substances for application in food or pharmaceutical products. ${ }^{12}$ Oil bodies, or oleosomes, are specialized organelles ubiquitously present in plant oil seeds as lipid storage compartments consisting of a hydrophobic core of triacylglycerol (TAGs), with a surrounding monolayer of phospholipids (PLs) where proteins, known as oleosins, with a size ranging from 0.5 to $2 \mu \mathrm{m}$ are present. ${ }^{13}$

Coconut (Cocos nucifera L.) milk present in its plant seed could serve as an important source of oil bodies that may be used as an encapsulating agent for various materials. ${ }^{14}$ It also possesses several other benefits such as its antibacterial, antiviral, and anti-inflammatory properties. Also, its ability to reduce cholesterol levels is an advantage over the commercially used liposome. ${ }^{15}$

Hence, coconut oil bodies (COB) which contains triacylglycerol and phospholipids could be a suitable candidate as drug carrier that could perform as well as liposomes. An application of $\mathrm{COB}$ as an alternative drug delivery system for anticancer drugs such as doxorubicin and paclitaxel would be better evaluated and characterized in this study.

\section{METHODS}

\section{Standards, Reagents, and Chemicals}

The chemicals and reagents used were $7 \mathrm{M}$ urea, hexane, bromothymol blue, Folin-Ciocalteu reagent, $0.1 \mathrm{M} \mathrm{HCl}, 0.1$ $\mathrm{M} \mathrm{NaOH}, 10 \% \mathrm{Na}_{2} \mathrm{CO}_{3}, \mathrm{NaCl}$, buffer 1 (10 mM sodium phosphate buffer $\mathrm{pH} 7.4$ with $0.4 \mathrm{M}$ sucrose), buffer 2 (5 $\mathrm{mM}$ sodium phosphate buffer $\mathrm{pH} 7.4$ with $0.2 \mathrm{M}$ sucrose and $0.1 \%(\mathrm{v} / \mathrm{v})$ Tween 20$)$, buffer 3 (10 mM sodium phosphate buffer $\mathrm{pH} 7.4$ with $0.25 \mathrm{M}$ sucrose and $2 \mathrm{M}$ sodium chloride), and sodium phosphate buffer saline ( $\mathrm{pH} 7.4$ and $\mathrm{pH}$ 5.0). All reagents used in this experiment were analytical grade and purchased from Sigma-Aldrich, USA.

\section{Isolation and Purification of COB from Cocos nucifera $L$.}

Using a sucrose gradient method done in a study by Santiago \& Devanadera $(2016)^{16}$, COB was isolated from the endosperm of Cocos nucifera $\mathrm{L}$ obtained from a local public market. Sucrose gradient was used for separating cell organelles, cytosolic or nuclear proteins from crude cellular extracts. Then, in a centrifuge tube, a 1:1 ratio of crude $\mathrm{COB}$ and buffer 1 were mixed and subjected to floatation centrifugation using a Heraeus Megafuge $8 \mathrm{R}$ centrifuge machine at $4^{\circ} \mathrm{C}, 10,000 \mathrm{x}$ g for 30 minutes. A solid oil pad was collected, transferred to a clean tube, heated, and mixed to homogenize the sample when added to buffer 2 . The procedure was repeated on oil pad for four times each with different reagents added accordingly - buffer 3, $7 \mathrm{M}$ urea, 10 $\mathrm{mM}$ sodium phosphate buffer ( $\mathrm{pH}$ 7.4), and hexane. Lastly, $\mathrm{COB}$ was washed with $10 \mathrm{mM}$ sodium phosphate buffer 
( $\mathrm{pH}$ 7.4) to remove any potentially toxic compounds such as the organic reagents used in the sample. Purity of $\mathrm{COB}$ was determined under microscope, wherein, the protein oleosin was clearly removed (bright field microscope) and the layer of oil body had become thinner (fluorescence microscope). The purified $\mathrm{COB}$ was stored at $4^{\circ} \mathrm{C}$ until further use.

\section{Microencapsulation of $\mathrm{COB}$ and Liposome}

\section{Oil Bodies Encapsulation}

An $800 \mathrm{uL}$ COB was sonicated for 10 minutes for complete disassembly into smaller particles or globules. ${ }^{16}$ In a separate tube, DOX (doxorubicin $\mathrm{HCl}$, Adriamycin ${ }^{\circledR}$, West-Ward Pharmaceuticals, USA) with $2 \mathrm{mg} / \mathrm{mL}$ dissolved in NSS solution and PTX (paclitaxel, Taxol ${ }^{\circledR}$, Bristol-Myers Squibb, USA), $800 \mu \mathrm{L}$ was mixed with $\mathrm{COB}$ and stored at $4^{\circ} \mathrm{C}$ for 10 minutes followed by thawing at room temperature. The freeze - thawing procedure was repeated thrice, after which the solution was left at room temperature for 1 hour to allow self-assembly. The solution was centrifuged at $10,000 \mathrm{x} \mathrm{g}, 4^{\circ} \mathrm{C}$, for 30 minutes. After centrifugation, the liquid supernatant was discarded and the oil pad containing the encapsulated DOX and PTX was collected. Samples were then lyophilized. After freeze-drying for 1 day, samples were stored at $-20^{\circ} \mathrm{C}$ freezer until further use. ${ }^{16}$

\section{Liposome Encapsulation}

Procedures on rehydrating and encapsulation using Liposome (Liposome kit AR grade, Sigma-Aldrich, USA) was done by mixing $0.85 \mathrm{~mL}$ distilled water with liposome for 30 secs. A $0.15 \mathrm{~mL}$ of $1 \mathrm{mg} / \mathrm{mL}$ DOX or $6 \mathrm{mg} / \mathrm{mL}$ PTX (separate from one another) was added and mixed, resulting into a homogenous milky suspension. Samples were then lyophilized. After freeze-drying for 1 day, samples were stored at $-20^{\circ} \mathrm{C}$ refrigerator until further use. ${ }^{16}$

\section{Determination of Encapsulation Efficiency (\%)}

A subsequent weighing was done with the following: empty tube, a tube with COB or liposome, and a tube with the encapsulated drug. COB-based drugs were centrifuged for $10000 \mathrm{x} \mathrm{g}, 30$ minutes at $4^{\circ} \mathrm{C}$, forming an oil pad which was collected and transferred to a new weighed empty tube while the addition of liposome, drugs (DOX \& PTX) was done for liposome-encapsulated drugs. To compute for the percentage of encapsulation efficiency of the wall materials, we used the formula: $\mathrm{EE} \%$ = (weight of drug in the capsule / weight of drug introduced) $\mathrm{x} 100$.

\section{Lipid Profiling of Samples}

\section{Fourier-Transform Infrared Spectroscopy (FTIR) Analysis}

FTIR for drug-loaded $\mathrm{COB}$ and liposome was obtained in the range of 4,000 to $400 \mathrm{~cm}^{-1}$ with IRAffinity-1S
FTIR Spectrometer (Shimadzu) to identify the functional groups of the samples and to determine the successful incorporation of the drugs into the coconut oil bodies.

\section{Gas Chromatography - Flame Ionization Detector (GC-FID) Analysis}

$\mathrm{COB}$ sample was submitted and analyzed using a Dani Master Fast GC in SentroTek to determine the fatty acid content. Samples were filtered into a sample vial using Whatman No. 1 and were placed on an autosampler injector. The injector was set at $250^{\circ} \mathrm{C}$ and the interface temperature at $270^{\circ} \mathrm{C}$ while the column was set at $50^{\circ} \mathrm{C}$ to $150^{\circ} \mathrm{C}$ with $15^{\circ} \mathrm{C} /$ minute temperature increments and $150^{\circ} \mathrm{C}$ to $250^{\circ} \mathrm{C}$ with $3^{\circ} \mathrm{C} /$ minute temperature increments. The column used Capillary GC column, SP-2560, Sigma-Aldrich. The gas pump was set at $38.0 \mathrm{~cm} /$ minute using hydrogen gas. Sulpeco 37 component fatty acid methyl esters (FAME) Mix (Sigma-Aldrich) was used as the FAME standard for GC analysis of the samples. The identity of the fatty acids was based on the peaks of samples related to the peaks of standards fatty acids in FAME mixture in the gas chromatograph. The quantitative data (percent fatty acid composition) were based on the peak area of the sample divided by the total peak area multiplied by 100 .

\section{pH Stability Studies}

COB DOX, COB PTX, Lipo DOX, and Lipo PTX were suspended in a PBS buffer ( $\mathrm{pH} 7.4$ and $\mathrm{pH}$ 5.0) for 30 days. Both initial and final absorbance of the samples were read in triplicates at 480nm (DOX) and 227nm (PTX) using a UV-VIS spectrophotometer. ${ }^{16}$

\section{In Vitro Drug Release Test}

Approximately $20 \mathrm{mg}$ and $60 \mathrm{mg}$ of DOX and PTX were weighed respectively, and each suspended separately in a $10 \mathrm{~mL}$ PBS buffer (pH 5.0 and 7.4 for both drugs) incubated at a constant temperature of $37^{\circ} \mathrm{C} \pm 1^{\circ} \mathrm{C}$. At the pre-determined time interval (per hour for 5 hours and after 20 hours incubation), $200 \mathrm{uL}$ aliquots were withdrawn from the dissolution medium and replaced with fresh PBS buffer to maintain constant volume and sink conditions. Samples were then analyzed at $480 \mathrm{~nm}$ (DOX) and $227 \mathrm{~nm}$ (PTX) using Multiskan GO UV-Vis spectrophotometer. All measurements were carried out three times. To calculate each sample concentration, DOX standard curve was constructed by serially diluting $25 \mathrm{uL}$ of $2 \mathrm{mg} / \mathrm{mL}$ DOX stock solution in a $50 \mathrm{uL}$ PBS buffer $(\mathrm{pH}$ 7.4) with a dilution factor of 1:2. A PTX standard curve was also constructed by serially diluting $12.5 \mu \mathrm{L}$ of $6 \mathrm{mg} / \mathrm{mL}$ PTX stock solution in a 15uL PBS buffer ( $\mathrm{pH}$ 7.4), and a $10 \mathrm{uL}$ Folin-Ciocalteu dye with a dilution factor of 1:2. Since concentration is an intrinsic property, $\mathrm{pH}$ condition of the buffer is negligible. Cumulative amount of PTX and DOX was calculated and expressed as percentage in terms of the mean obtained from PTX and DOX standard curve. 


\section{Determination of cholesterol}

The cholesterol level on the sample was determined based on the standard protocol of Total Cholesterol Kit (Human Diagnostics, Germany). A $1 \mu \mathrm{L}$ of cholesterol standard was placed in three wells of a 96-well plate and were added with a reagent buffer. Then, as an initial absorbance, cholesterol standard was mixed with the reagent buffer and then incubated for 10 minutes at $37^{\circ} \mathrm{C}$ and measured using UV$V$ is spectrophotometer at $500 \mathrm{~nm}$. Thereafter, samples such as COB-DOX, COB-PTX, Lipo-DOX, and Lipo-PTX were mixed with reagent buffer, incubated for 10 minutes at $37^{\circ} \mathrm{C}$, and measured using UV-Vis spectrophotometer at $500 \mathrm{~nm}$. Samples were carried out in triplicates. A cholesterol standard curve was constructed by serial dilution of $200 \mathrm{mg} / \mathrm{dL}$ stock solution of cholesterol standard, with a dilution factor 1:2.

\section{Data analysis}

In this study, all data were expressed as mean \pm standard deviation $(n=3)$. Statistical analyses were performed using Microsoft Excel 2010. A paired t-test was used to determine the significant difference in the experimental method for $\mathrm{pH}$ stability study and cholesterol-lowering determination while one-way ANOVA $(p<0.05)$ was used for the in vitro drug release.

\section{RESULTS AND DISCUSSION}

\section{Drug Microencapsulation}

Microencapsulation is a rapidly growing technique of novel drug delivery system which purportedly enhances the therapeutic efficacy of conventional drugs. ${ }^{15}$ Success in drug formulation heavily relies on the chemical nature of drug microencapsulation as it will help to address the issues in drug loading and encapsulation efficiency. ${ }^{17}$ Encapsulation efficiency is the percentage of drug molecules successfully entrapped into its drug carrier. Drug loading is regarded as a key and critical step in characterizing the quality of the formulation. Thus, to achieve a high encapsulation efficiency, factors involving the type, size, surface charge, rigidity, and method of preparation for the drug carrier are highly considered. Moreover, the characteristics and complexing agents of the drug to be encapsulated are considered as well. ${ }^{18}$

Currently, liposome is still widely used globally as a versatile drug carrier capable of encapsulating varying lipophilicities of drugs, in such cases a hydrophilic drug can be found in the inner aqueous phase. On the other hand, a hydrophobic drug can be encapsulated into the phospholipid bilayers. ${ }^{19}$ However, with a hydrophobic domain, liposome can only accommodate a limited quantity of drug. These led to a low drug/drug carrier ratio since the drug has a low affinity to the phospholipid bilayer, thus supporting the $71 \%$ encapsulation efficiency of Lipo-PTX. The low affinity of PTX in the phospholipid bilayer can be attributed to the natural high cholesterol content of the liposome formulation used. High content of cholesterol reduces the penetration of PTX into the lipid bilayer due to decreased flexibility of the membrane. In addition, the low encapsulation efficiency of PTX to liposome is inherently due to lipophilicity of the drug molecule which competes with cholesterol in occupying the hydrophobic space designed for aqueous solutions into the lipid bilayer. ${ }^{20}$

Lipo-DOX yields $85 \%$ encapsulation efficiency because of its biocompatibility with the drug carrier and drug, being hydrophilic in nature. A liposomal DOX obtained a maximum encapsulation efficiency nearly $100 \%$ using a $\mathrm{pH}$ gradient technique, but a few modifications had been applied by adding $5 \%$ and $10 \%$ palm oil in the liposomal formulations through a freeze-thaw method. ${ }^{21}$ Doxorubicin could self-associate in varying concentrations of buffers, $\mathrm{pH}$ and other experimental conditions. It favors assembling interactions with the hydrophobic space of the lipid bilayer of the liposome which is of high aqueous solubility conditions. However, the DOX is too sensitive to aqueous dispersal of liposome rendering it unstable upon drug release as can be seen in well-documented Lipo-DOX extravasation of capillaries and fenestrations in the cells and tissues of circulation. ${ }^{22}$ Lipo-DOX had been a successful and perfect drug formulation for cancer therapy; a 100\% encapsulation efficiency can possibly be achieved with just appropriate conditions, modifications, and methods used.

COB encapsulated drugs showed higher encapsulation efficiency compared to liposome encapsulated drugs and PTX encapsulated in $\mathrm{COB}$ have an approximate encapsulation efficiency of $100 \%$ due to the possibility that excess PTX and $\mathrm{COB}$ globules are present in the solution. In this study, an assumption was proposed that since PTX is hydrophobic in nature, it completely solubilizes into the matrix of $\mathrm{COB}$ containing a high lipid content, (ie, they share similar nonaqueous solubility property). The absence of cholesterol can also be considered as a key factor in the potential complete penetration of PTX in COB. Coconut oil is $90 \%$ saturated fats and $10 \%$ unsaturated fats. ${ }^{23}$ PTX may adhere to the predominant saturated fatty acid portions of $\mathrm{COB}$ to strengthen its encapsulation efficiency. This structuration of COB is more helpful in the case of entrapment of DOX as it will generate a tight aqueous center of $\mathrm{COB}$ which mimics the physicochemical nature DOX. Nevertheless, such assumption must be further investigated.

\section{Drug Encapsulation Analysis by FTIR Spectroscopy}

FTIR is a powerful and versatile analysis tool in the pharmaceutical industry for its structural elucidation, drug formulation development, and validation. Qualitatively, FTIR identifies chemical bond functional group by their characteristic absorption in the infrared region due to its vibrational change from a ground state to an excited state. ${ }^{24}$ FTIR identified the functional groups and confirmed incorporation of the DOX and PTX into the $\mathrm{COB}$ and liposomes. The representative spectra of $\mathrm{COB}$ exhibited 
absorption peaks located at 2922, 1155 and $720 \mathrm{~cm}^{-1}$. The peaks at $2900 \mathrm{~cm}^{-1}$ confirmed the presence of the $\mathrm{CH}_{3}$ (alkanes) groups. The peak found in $1100 \mathrm{~cm}^{-1}$ represented $\mathrm{a}=\mathrm{C}-\mathrm{H}$ bend. The peak at $700 \mathrm{~cm}^{-1}$ confirmed the presence of the $\mathrm{CH}_{2}$ stretch in the $\mathrm{COB}$ analyzed. Anthacycline are compounds of a glycoside structure, containing a sugar and aglycone moiety. The sugar is usually a daunosamine whereas the aglycone moiety consists of four six-carbon rings. The structure of DOX consists of a hydrophobic anthracycline backbone, along with several active sites, including an amine, ketone, and hydroxyl groups. The absorption peaks were located at $3355,1742,1638$, and $687 \mathrm{~cm}^{-1}$; this can be observed in Figure 1.

The broad band contour which appears in the range of $3000-3400 \mathrm{~cm}^{-1}$ is related to $\mathrm{OH}$ or $\mathrm{NH}_{2}$ stretch. The $\mathrm{OH}$ absorptions are generally quite intense and smoothly curved, whereas $\mathrm{NH}_{2}$ stretches are weaker and narrower depending on whether it is primary, secondary, or tertiary amine. The absorption peak at $1700 \mathrm{~cm}-1$ can be attributed to the
$\mathrm{C}=\mathrm{O}$ (ketone and aldehyde) groups. Since DOX also has a benzene ring, then a characteristic absorption at about $680-900 \mathrm{~cm}^{-1}$ can be observed. The interaction between a drug and drug carrier can also be predicted by using FTIR. In Figure 1, the characteristic of Lipo-DOX shows all the band characteristic of DOX displayed in the figure but without any significant peak shift.

FTIR spectra of $\mathrm{COB}$ shows a strong correlation to the results obtained by GC-FID analysis, in which there is a high content of saturated fatty acids. Saturated fatty acid structurally consists of alkanes and carboxyl constituents. The typical absorption peak of $\mathrm{CH}_{2}$ stretching vibrations is at $2922 \mathrm{~cm}^{-1}$. Likewise, a peak in the $1700 \mathrm{~cm}^{-1}$ can be observed for carboxyl constituent. Other distinct peaks at $1155 \mathrm{~cm}^{-1}$ and $1250 \mathrm{~cm}^{-1}$ corresponds to $=\mathrm{C}-\mathrm{H}$ and $\mathrm{C}-\mathrm{C}$ bending vibrations, respectively.

Since paclitaxel consists of taxane ring with fourmembered oxetane side ring and an active homochiral ester side chain, absorption peaks can be found at 3355,1742 , and

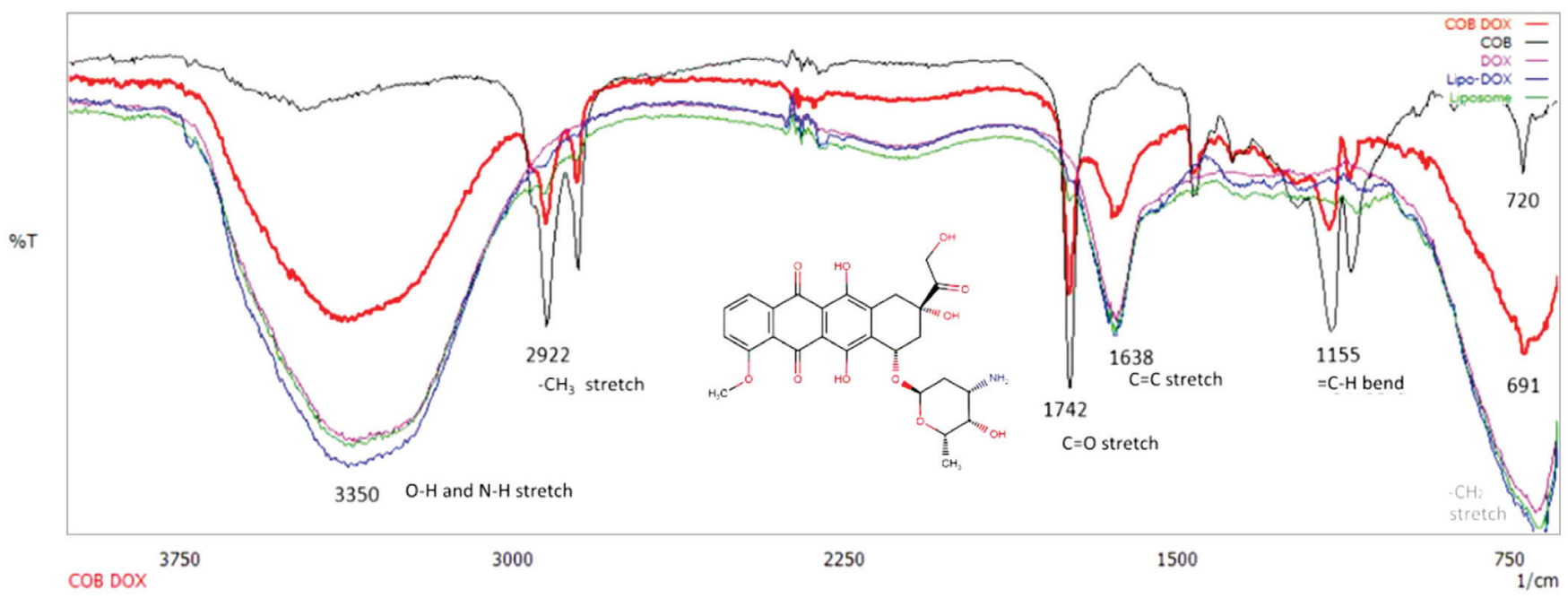

Figure 1. FT-IR absorption spectra DOX-based encapsulation using $\mathrm{COB}$ and Liposome.

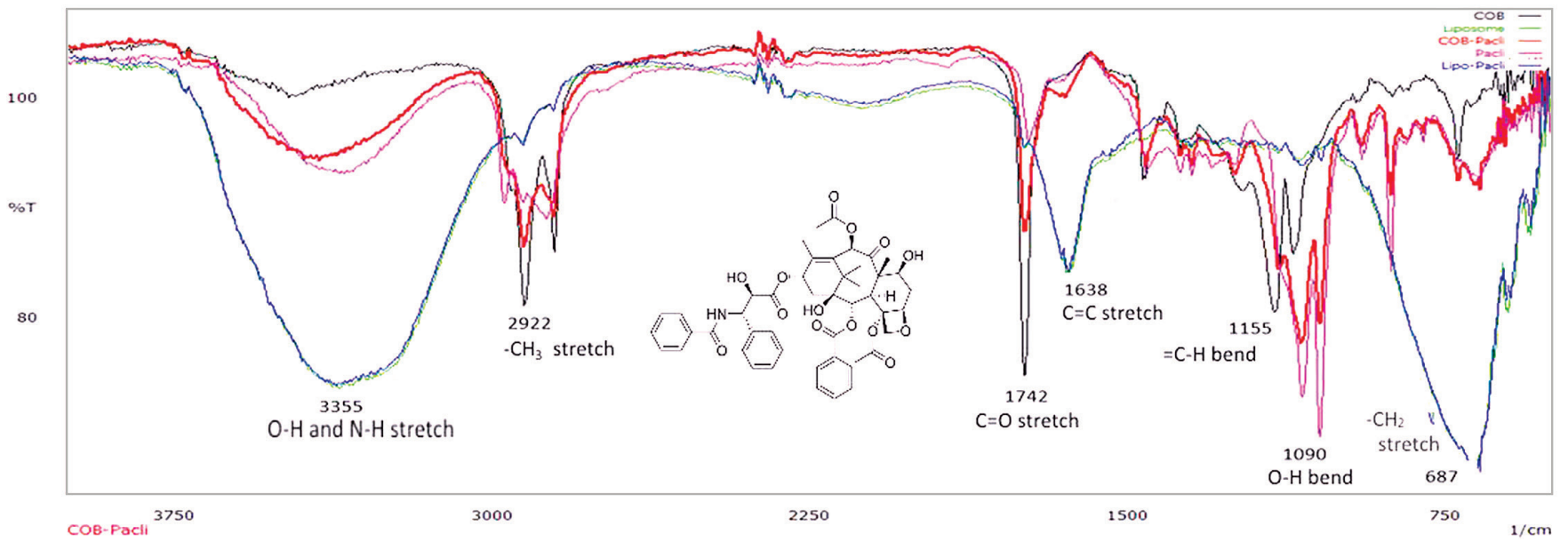

Figure 2. FT-IR absorption spectra PTX-based encapsulation using COB and Liposome. 
$687 \mathrm{~cm}^{-1}$. An absorption peak at $1638 \mathrm{~cm}^{-1}$ indicates aromatic ring, stretch $\mathrm{C}=\mathrm{C}$, an $\mathrm{O}-\mathrm{H}$ at $3355 \mathrm{~cm}^{-1}$, and $\mathrm{C}=\mathrm{O}$ for the ester group at $1742 \mathrm{~cm}^{-1}$ as shown in Figure 2. More importantly, PTX is preferentially favorable for COB than liposome since all characteristic band of the PTX is displayed, and no spectral shift is observed (Figure 2).

\section{Analysis of Fatty Acid Composition of COB}

Coconut oil bodies are rich source of medium chain fatty acids (MCFA). MCFA comprised of lauric, myristic, caprylic, capric, and caproic fatty $\mathrm{acid}^{24}$ which was further verified on the gas chromatography $(\mathrm{GC})$ data on the fatty acid composition of coconut oil bodies as seen on Figure 3. In agreement to the literature, $\mathrm{GC}$ analysis of $\mathrm{COB}$ revealed almost $50 \%$ lauric acid, followed by small amounts of myristic acid, caprylic acid, and palmitic acids as seen in Figure 3. It also conforms to the presence of about 10\% unsaturated fatty acids such as oleic and linoleic acid. ${ }^{25,26}$ This analysis further confirms our assumption on the presence of small amount of cholesterol in $\mathrm{COB}$ which allows for stronger adherence and penetration of DOX and PTX into the COB. MCFA allows flexible structuration of COB in the encapsulation of chemotherapeutic drugs to prevent it from rapid dispersal and dissolution both in the aqueous and lipophilic parts of the circulation. Utilization of $\mathrm{COB}$ formulation provides nutritional benefits and a number of pharmacological benefits of MCFA, particularly in cardioprotection and improvement of hepatic function. ${ }^{27}$

\section{pH Stability Studies}

Stability testing is a crucial part of drug formulation since it ensures quality, efficacy, and safety of a drug and considered by the Food and Drug Administration (FDA) to be a prerequisite for a pharmaceutical product to be sold in the market. For a pharmaceutical product to be stable, it must retain its properties and characteristics possessed within a specified limit, throughout its period of storage and use. ${ }^{28}$ Also, it must be evaluated for numerous stressors including temperature, oxidation, UV light exposure, and hydrolysis at different $\mathrm{pH}$. The drug content of COB-DOX between

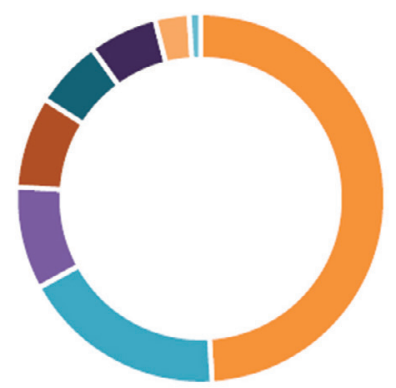

- Lauric Acid " Myristic Acid " Caprylic Acid " Palmitic Acid - Oleic Acid " Capric Acid " Stearic Acid " Linoleic

Figure 3. Fatty acid composition of coconut oil bodies. freshly prepared (initial day) and after storage at a room temperature for 30 days were measured by reading their absorbance at $480 \mathrm{~nm}$. An increase in absorbance from initial drug release until 30 days of incubation indicates the slow degradation of the $\mathrm{COB}$ and the slow release of DOX. The gradual release of DOX from the capsule causes relevant increase in the absorbance as portion of the drug molecule, particularly the ring structures that contain resonance, can now interact and absorb light significantly. Figure 4 clearly shows that the absorbance readings of COB-DOX initial and COB-DOX final are significantly different $(p<0.05)$ and had increased. The results indicate that the COB-DOX are stable even at 30 days incubation. On the other hand, COB-PTX's absorbance reading is not within the range of the standard curve generated.

Also, in Figure 4, there is a significant difference ( $\mathrm{p}<$ 0.05 ) between the concentration of COB-DOX in a neutral and acidic condition. At acidic condition, hydrolysis of the coating material of COB-DOX will be favored resulting to more DOX molecules escaping the drug carrier. The acid environment gradually disrupts the quenching of $\mathrm{COB}$ and DOX molecules resulting to higher absorbance reading. Quenching is a type of complexation reaction where an active drug or molecule binds to its encapsulating material or carrier which loses its ability to absorb light in aqueous solution. A recent study by DebMandal $\mathrm{M}$ and Mandal S, suggests that the slight increase of absorbance in acidic environment may be associated to deglucosaminyl doxorubicin (A-I) - one of the degradation products of doxorubicin when exposed to acidic solutions for long time. A-I, is formed by the loss of the glucosamine moiety due to cleavage of glycosidic linkage between the tetracycline ring and the glucosamine moiety, which has the following proposed mass fragmentation product ions and $\mathrm{m} / \mathrm{z}$ ratio (397.0914,379.0824,361.0781, and 321.0745) as determined by their LC-MS-TOF analysis. ${ }^{27,28}$

\section{In Vitro Release of Encapsulated Drugs}

Characterization of encapsulation efficiency and in vitro drug release are known to be a key performance parameter

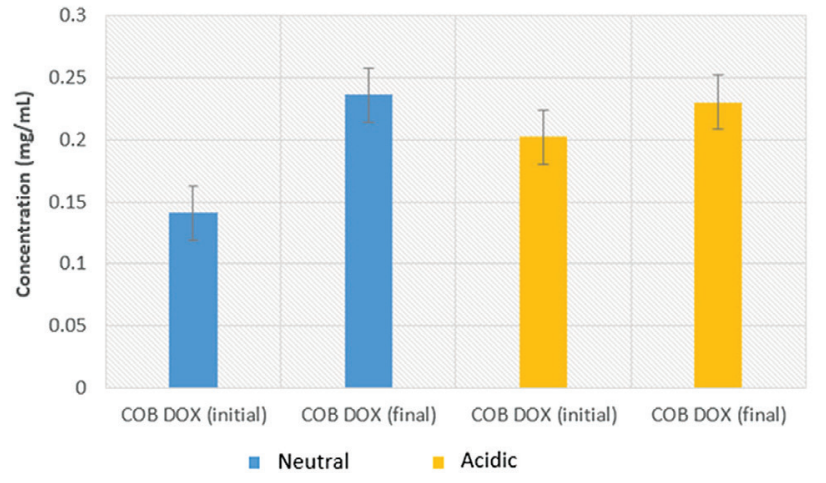

Figure 4. $\mathrm{pH}$ stability profile of COB DOX in an acidic (blue) and neutral (yellow) condition. 
for drug formulation and product quality. These can also predict the in vivo performance of the encapsulated drugs. In fact, in vitro drug release is a process that induces drug release through either of these three mechanisms (1) erosion of the matrix to release drug from the core, (2) subsequent diffusion of drug molecules out the microparticle matrix, and (3) desorption of weakly bound drug molecule to the surface of the microparticle. Usually, a rapid initial release is attributed to the mechanism where a weakly bound drug is adsorbed only to a surface of the microparticle instead of being incorporated inside the polymer microparticle. ${ }^{29}$

In understanding the drug release mechanism, factors such as drug adsorption in the membrane/polymer of a drug carrier, surface-area-to-volume ratio of drug carrier, drug solubility, intermolecular interaction between the drug, and matrix of drug carrier greatly influenced drug's pharmaceutical and clinical performance. ${ }^{30,31}$ The release rate was expressed using a zero-order kinetic model, wherein concentration is independent of time. The following formulas were used: Amount of drug $=$ Concentration $(\mathrm{mg} / \mathrm{mL}) \mathrm{x}$ dissolution bath $(\mathrm{mL})$ and $\mathrm{CDR}(\%)=(($ Amount of drug $\mathrm{n}+($ Amount of drug N*dilution factor)) / (Total drug(mg)) $\mathrm{x} 100$ wherein $\mathrm{N}$ represents the amount of successive drug; $\mathrm{n}$ represents the amount of preceding drug; $\mathrm{CDR} \%=$ percentage of cumulative drug release.

At $\mathrm{pH} 7.4$, the initial release rate of Lipo-DOX was slow and cumulative release were $13.2 \%, 18.5 \%$, and $26.2 \%$ for the first 3 hours, respectively, and $17.1 \%$ for the $24^{\text {th }}$ hour (Figure 5). When the $\mathrm{pH}$ value is slightly acidic (5.0), the cumulative releases were $10.6 \%, 26.5 \%$, and $29.3 \%$ for the first 3 hours, respectively and $11.6 \%$ for the $24^{\text {th }}$ hour (Figure 5). Liposomes' phospholipid bilayer and cholesterol composition might have maintained a tight and compact structure and entrapped the DOX molecules in the core, preventing its release into the acidic and neutral environment, thus a low percentage with a range of $10-63 \%$ is observed,

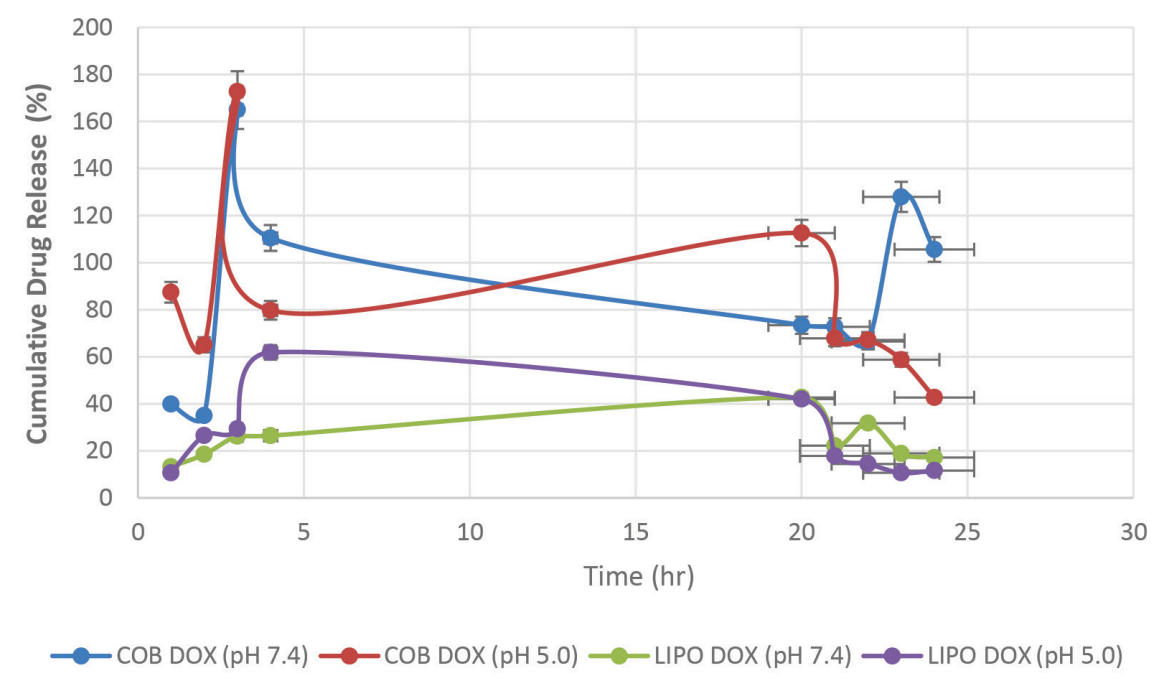

Figure 5. In vitro DOX release profiles from $\mathrm{COB}$ and liposome at $\mathrm{pH} 7.4$ and 5.0.

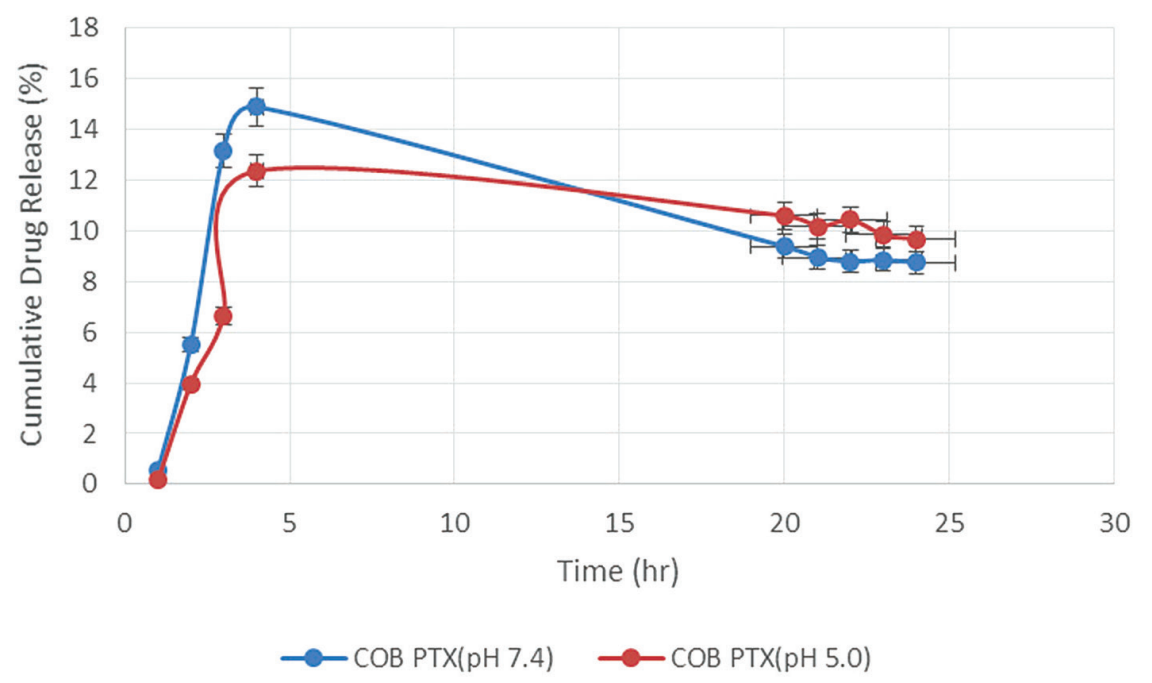

Figure 6. In vitro PTX release profiles from $\mathrm{COB}$ and liposome at $\mathrm{pH} 7.4$ and 5.0. 
compared to $\mathrm{COB}$ encapsulated drug. The DOX release from $\mathrm{COB}$ in vitro in $\mathrm{PBS} \mathrm{pH} 5.0$ is faster than $\mathrm{pH} 7.4$ as presented in Figure 5.

Moreover, after a few hours, all drug molecules had been released in the medium. As shown in Figure 5, the percentage difference of cumulative drug release is due to not uniform preparation of drug molecules present in the original sample. Within 3 hours, 7\% of PTX is released in the acidic medium while $13 \%$ is released in the neutral medium (Figure 6). Theoretically, COB-PTX should have released higher concentrations in an acidic condition than in a neutral condition since paclitaxel consists of ionizable groups which are capable of being easily degraded but the study had proposed that $\mathrm{COB}$ remained intact at acidic condition and kept PTX enclosed in its matrix. Overall, both liposome-encapsulated drugs and $\mathrm{COB}$-encapsulated drugs exhibited a biphasic release profile with an initial burst within 3 hours followed by a sustained, plateau drug release until 24 hours.

\section{Cholesterol Level Determination on the Encap- sulating Material}

Since 1961, the American Heart Association and the United States Dietary Guidelines have recommended reduction of dietary saturated fats because of its potential risk in the incidence of developing atherosclerosis and cardiovascular disease (CVD). In lieu, saturated fats were replaced by polyunsaturated and monounsaturated fats. The study is concerned on possible side effects of the $\mathrm{COB}$ which must be addressed and investigated. As seen in Figure 7, there is a significant difference $(\mathrm{p}<0.05)$ between the final concentration and initial concentration of both COB-DOX and COB-PTX. The observed difference in cholesterol concentration of COB-DOX is due to biphasic system for hydrophobic interactions in an amphiphilic environment. Biphasic system accounts for doxorubicin's partitioning because the drug is present at its cation form which strongly

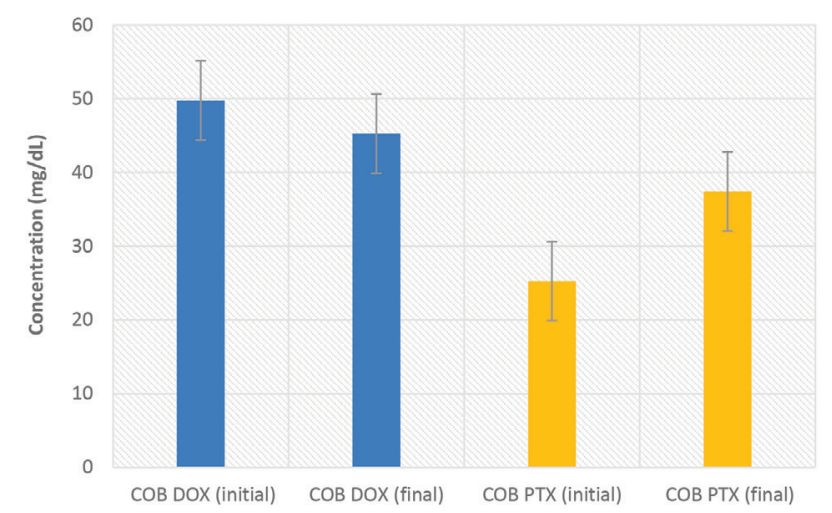

Figure 7. Comparison of cholesterol concentration of coconut oil bodies in two drugs, Doxorubicin (DOX,blue) and Paclitaxel (PTX, yellow) before and after microencapsulation. encourages the saturated fatty acids of $\mathrm{COB}$ to straighten, further ordering and rigidifying the encapsulated drug with lesser cholesterol molecules. ${ }^{28,32,33}$ The observed increase in cholesterol concentration for COB-PTX suggests medium chain and long chain fatty acids promote formation of multilamellar vesicles where cholesterol positions itself establishing electrostatic and hydrophobic interactions with PTX. It will induce fluidization mainly by cholesterol, thus, improving the accommodation and stability of incorporation PTX to COB. ${ }^{34}$

The process of microencapsulation using natural coconut oil bodies incorporated in these chemotherapeutic drugs has not only decreased cholesterol levels but also make the drug more stable with a drug release profile showing a rapid outburst within 3 hours. The studied physicochemical properties of $\mathrm{COB}$ as encapsulating agent for DOX and PTX warrants further investigation to validate its usefulness and efficacy as drug carrier of cancer chemotherapeutic agents.

\section{CONCLUSION}

Coconut oil bodies (COB) showed promising results as an encapsulating material for doxorubicin as shown by the high encapsulation efficiency exhibited. Incorporation of the drug within the wall of encapsulating material was also confirmed by the FTIR results. Both FTIR and GC-FID had validated and provided the fatty acid profile required to understand other steps of drug formulation. The stability of the $\mathrm{COB}$ was found to last less than 30 days and it releases DOX more favorably in an acidic environment which suggests ideal targeted release in cancer cells. However, no assurance is yet implied for paclitaxel, thus further optimization for its drug formulation is proposed. Overall, $\mathrm{COB}$ performs well and displays great potential as an alternative wall material for the currently used liposome.

\section{Statement of Authorship}

All authors participated in data collection, analysis, written reports, and approval of the final version of the manuscript.

\section{Author Disclosure}

All authors declared no conflicts of interest.

\section{Funding Source \\ None.}

\section{REFERENCES}

1. Vasir JK, Labhasetwar V. Targeted drug delivery in cancer therapy. Technol Cancer Res Treat. 2005 Aug; 4(4):363-74. doi: 10.1177/153303460500400405.

2. Beslija S, Bonneterre J, Burstein H, Cocquyt V, Gnant M, Goodwin $\mathrm{P}$, et al. Second consensus on medical treatment of metastatic breast cancer. Ann Oncol. 2007 Feb; 18(2):215-25. doi: 10.1093/annonc/ mdl155. 
3. Doxorubicin. Johnson-Arbor \& Dubey. In: StatPearls. Treasure Island (FL): StatPearls Publishing; 2018.

4. O'Brien KM, Dirmeier R, Engle M, Poyton RO. Mitochondrial protein oxidation in yeast mutants lacking manganese-(MnSOD) or copperand zinc-containing superoxide dismutase (CuZnSOD): evidence that $\mathrm{MnSOD}$ and $\mathrm{CuZnSOD}$ have both unique and overlapping functions in protecting mitochondrial proteins from oxidative damage. J Biol Chem. 2004 Dec; 279(50):51817-27. doi: 10.1074/jbc.M405958200.

5. Surapaneni MS, Das SK, Das NG. Designing Paclitaxel drug delivery systems aimed at improved patient outcomes: current status and challenges. ISRN Pharmacol. 2012; 2012: 623139. doi: 10.5402/2012/623139.

6. Tiwari G, Tiwari R, Sriwastawa B, Bhati L, Pandey S, Pandey P, et al. Drug delivery systems: An updated review. Int J Pharm Investig. 2012 Jan; 2(1):2-11. doi: 10.4103/2230-973X.96920.

7. Silva P, Fries L, Menezes C, Holkem A, Schwan C, Wigmann E, et al. Microencapsulation: concepts, mechanisms, methods, and some applications in food technology. Ciência Rural. 2014 Jul; 44(7): 1304-11. doi: 10.1590/0103-8478cr20130971

8. Tomaro-Duchesneau C, Saha S, Maholtra M, Kahouli I, Prakash S. Microencapsulation for the therapeutic delivery of drugs, live mammalian and bacterial cells, and other biopharmaceutics: current status and future directions. J Pharm (Cairo). 2013; 2013:103527. doi: $10.1155 / 2013 / 103527$

9. Tsai W, Rizvi S. Liposomal microencapsulation using the conventional methods and novel supercritical fluid processes. Trends Food Sci Technol. 2016 Sep; 55:61-71. doi:10.1016/j.tifs.2016.06.012

10. Sercombe L, Veerati T, Moheimani F, Wu SY, Sood AK, Hua S. Advances and challenges of liposome assisted drug delivery. Front Pharmacol. 2015; 6:286. doi: 10.3389/fphar.2015.00286

11. Allen T, Cullis P. Liposomal drug delivery systems: from concept to clinical applications. Adv Drug Deliv Rev. 2013 Jan; 65(1):36-48. doi: 10.1016/j.addr.2012.09.037.

12. Acevedo F, Rubilar M, Jofre I, Villaroel M, Navarrete P, Esparza $\mathrm{M}$, et al. Oil bodies as a potential microencapsulation carrier for astaxanthin stabilization and safe delivery. J Microencapsul. 2014; 31(5): 488-500. doi: 10.3109/02652048.2013.879931.

13. Bonsegna S, Bettini S, Pagano R, Zacheo A, Vergaro V, Giovinazzo G, et al. Plant oil bodies: Novel carriers to deliver lipophilic molecules. Appl Biochem Biotechnol. 2011 Mar; 163(6):792-802. doi: 10.1007/s12010-010-9083-0.

14. Lima EBC, Sousa CNS, Meneses LN, Ximenes NC, Santos Junior MA, Vasconcelos GS, et al. Cocos nucifera (L.) (Arecaceae): A phytochemical and pharmacological review. Braz J Med Biol Res. 2015 Nov; 48(11):953-64. doi: 10.1590/1414-431X20154773.

15. Venkatesan P, Manavalan R, Valliappan K. Microencapsulation: A vital technique in novel drug delivery system. J Pharm Sci Res. 2009 Jan; 1(4):26-35.

16. Santiago MR, Devanadera MKP. Microencapsulation of Pediococcus spp. with coconut oil bodies and oleosin for improved viability and enhanced targeted delivery in the gastrointestinal tract. Philipp Agric Scientist. 2016 Sep; 99(3):288-95.

17. Singh MN, Hemant KS, Ram M, Shivakumar HG. Microencapsulation: A promising technique for controlled drug delivery. Res Pharm Sci. 2010 Jul; 5(2):65-77.

18. Prasanth Kumar PK, Gopala Krishna AG. Physicochemical characteristics of commercial coconut oils produced in India. Grasas y Aceites. 2015 Jan-Mar; 66(1):e062. doi: 10.3989/gya.0228141
19. Li C, Li X, Li S, Weng Y, Wang K, Zhang T, et al. Development and validation of a method for determination of encapsulation efficiency of CPT- 11/DSPE-mPEG2000 nanoparticles. Med Chem. 2016; 6(5):345-8. doi:10.4172/2161-0444.1000368

20. Yang T, Cui FD, Choi MK, Lin H, Chung SJ, Shim CK, et al. Liposome formulation of paclitaxel with enhanced solubility and stability. Drug Deliv. 2007 Jul; 14(5):301-8. doi: 10.1080/10717540601098799.

21. Barenholz Y. Relevancy of drug loading to liposomal formulation therapeutic efficacy. J Liposome Res. 2003 Feb;13(1):1-8. doi: 10.1081/ lpr-120017482.

22. Abraham SA, Waterhouse DN, Mayer LD, Cullis PR, Madden TD, Bally MB. The liposomal formulation of doxorubicin. Methods Enzymol. 2005; 391:71-97. doi: 10.1016/S0076-6879(05)91004-5.

23. Eyres L, Eyres MF, Chisholm A, Brown RC. Coconut oil consumption and cardiovascular risk factors in humans. Nutr Rev. 2016 Apr; 74(4):267-80. doi: 10.1093/nutrit/nuw002.

24. Sabeti B, Noordin MI, Mohd S, Hashim R, Dahlan A, Javar HA. Development and characterization of liposomal doxorubicin hydrochloride with palm oil. Biomed Res Int. 2014;2014:765426. doi: 10.1155/2014/765426.

25. Chowdhury K, Banu LA, Khan S, Latif A. Studies on the fatty acid composition of edible oil. Bangladesh J Sci Ind Res. 2008 Feb; 42(3):311-6. doi: 10.3329/bjsir.v42i3.669

26. Rakesh P, Charmi P, Rajesh KS. Quantitative analytical applications of FTIR spectroscopy in pharmaceutical and allied areas. J Adv Pharm Edu Res. 2014 Apr-Jun; 4(2):145-57.

27. DebMandal M, Mandal S. Coconut (Cocos nucifera L.: Arecaceae): in health promotion and disease prevention. Asian Pac J Trop Med. 2011 Mar; 4(3):241-7. doi: 10.1016/S1995-7645(11)60078-3.

28. Boateng L, Ansong R, Owusu WB, Steiner-Aseidu M. Coconut oil and palm oil's role in nutrition, health and national development: A review. Ghana Med J. 2016 Sep; 50(3):189-96.

29. Bajaj S, Singla D, Sakhuja, N. Stability Testing of Pharmaceutical Products. J Appl Pharm Sci. 2012; 2(3):129-38. doi: 10.7324/JAPS. 2012.2322

30. Kaushik D, Bansal G. Four new degradation products of doxorubicin: An application of forced degradation study and hyphenated chromatographic techniques. J Pharm Anal. 2015 Oct; 5(5):285-95. doi: 10.1016/j.jpha.2015.05.003.

31. Wallace SJ, Li J, Nation RL, Boyd BJ. Drug release from nanomedicines: Selection of appropriate encapsulation and release methodology. Drug Deliv Transl Res. 2012 Aug; 2(4):284-92. doi: 10.1007/s13346-012-0064-4.

32. Alves AC, Magarkar A, Horta M, Lima JLFC, Bunker A, Nunes $C$, et al. Influence of doxorubicin on model cell membrane properties: insights from in vitro and in silico studies. Sci Rep. 2017 Jul;7(1):6343. doi: 10.1038/s41598-017-06445-z.

33. Róg T, Pasenkiewicz-Gierula M, Vattulainen I, Karttunen $M$. Ordering effects of cholesterol and its analogues. Biochim Biophys Acta. 2009 Jan; 1788(1):97-121. doi: 10.1016/j.bbamem.2008.08.022.

34. Hong SS, Choi JY, Kim JO, Lee MK, Kim SH, Lim SJ. Development of paclitaxel-loaded liposomal nanocarrier stabilized by triglyceride incorporation. Int J Nanomedicine. 2016 Sep; 11:4465-77. doi: 10.2147/IJN.S113723. 